\title{
Independence of question-answering strategy and searched representation
}

\author{
MURRAY SINGER \\ University of Manitoba, Winnipeg, Manitoba, Canada
}

\begin{abstract}
Because many studies of question answering, recall, and recognition have examined script-based messages, a spurious link between answering strategy and searched representation has emerged: Direct retrieval has been associated with the episodic representation of a discourse or a learned list, whereas plausibility judgment has been associated with general knowledge structures. Evidence of the independence of answering strategy and searched representation is presented. First, previous research and intuition both provide examples of the application of either strategy to either type of representation. Second, there is empirical evidence of the application of either strategy to both message text bases and general knowledge. Third, the effects of factors influencing strategy selection are independent of the type of searched representation. It is concluded that answering strategy is independent of the representation to which it is applied.
\end{abstract}

The study of the psychology of question answering has resulted in the identification of different answering strategies. In particular, it is possible either to directly retrieve a questioned idea from memory or to answer by evaluating the plausibility of the questioned idea (e.g., Reder, $1982,1987)$. The goal of this paper is to present evidence that question-answering strategy is independent of the type of knowledge structure to which the strategy is applied. For convenience, the target knowledge structure will be called the searched representation. It will be argued that, although the independence of answering strategy and searched representation is implicit in the psychological literature on question answering and sentence verification, the distinction between these dimensions has not been clearly drawn.

The paper will be structured as follows: First, the current status of the analysis of answering strategies will be briefly reviewed. A potential confounding between answering strategy and searched representation is considered. Next, some studies that have not confounded these two dimensions but have also not clearly distinguished them will be examined. Finally, the theoretical implications of the distinction between these two dimensions of question answering will be considered.

\section{Question-Answering Strategies}

The cognition of question answering has been carefully scrutinized during the past 15 years. The ability to answer questions about text has been identified as an essential criterion of comprehension, more important than successful paraphrasing or summarization (Lehnert, 1977).

This research was supported by Grant A9800 from the Natural Sciences and Engineering Research Council of Canada. Requests for reprints should be addressed to Murray Singer, Department of Psychology, University of Manitoba, Winnipeg, Manitoba R3T 2N2, Canada.
The quality and speed of people's answers to questions about familiar concepts and about stereotypical situations has been proposed to reveal the structure of the corresponding knowledge (e.g., Galambos \& Black, 1985; Graesser, Robertson, Lovelace, \& Swinehart, 1980).

An important development in this field has been the identification of numerous stages of mental processing that contribute to question answering (e.g., Graesser \& Murachver, 1985; Lehnert, 1977; Singer, 1986). Successful question answering has been proposed to require the parsing, propositional encoding, and conceptual categorization of the question, the search of memory, the comparison of the retrieved information with the questioned idea, and response formulation. This stagewise analysis has contributed to the identification of central problems of question answering, even though it oversimplifies the interactions between the stages.

Reder (1987) proposed that a processing stage of strategy selection precedes memory search in question answering. She presented evidence that one may either directly retrieve a questioned fact from memory or evaluate its plausibility with reference to existing knowledge. Suppose that one learns the following set of facts about Alan (Reder \& Anderson, 1980):

1. a. Alan arrived at the train station early.

b. Alan bought a ticket for the 10:00 train.

c. Alan heard the conductor call, "All aboard."

If subsequently asked, "Did Alan wait for the express on the platform?" one may attempt to directly retrieve the questioned idea from an episodic representation of the learned facts, such as a text base (Kintsch \& van Dijk, 1978). Alternatively, one may judge the plausibility of the question statement with reference to pertinent knowledge. For the present example, both the content of the learned facts and one's general knowledge about train 
travel might suggest that it is indeed plausible that the express was awaited on the platform.

Reder $(1982,1987)$ identified several principles of the selection of answering strategies. Some of these principles dispel prior misconceptions about the relationship between the retrieval and plausibility strategies. First, retrieval is not always more efficient than plausibility judgment. For example, in a delayed verification task, correct answer time was faster under instructions to use the plausibility strategy than direct retrieval (Reder, 1982). Direct retrieval is more efficient than plausibility judgment only when the fine details of the message are still available, shortly after encoding.

Second, the strategies may be mixed. For example, after failing to retrieve the idea that Alan waited for the express on the platform, one may proceed to evaluate its plausibility. A corollary of this principle is that direct retrieval does not always precede plausibility judgment (cf. Camp, Lachman, \& Lachman, 1980; Lehnert, 1977, p. 69). In this regard, Reder (1982) reported that, in immediate testing, answer time was faster under retrieval instructions than under plausibility instructions. This indicates that the plausibility participants, in keeping with their instructions, applied the plausibility strategy first. Had they tried direct retrieval first, it would have been successful, and answer times would have equaled those observed in the retrieval instruction condition.

A third principle is that answering-strategy selection is influenced by intrinsic and extrinsic question factors (Reder, 1982, 1987; Reder \& Anderson, 1980; Reder \& Ross, 1983; Reder \& Wible, 1984). Intrinsic factors include the familiarity and activation of the questioned idea. In particular, high familiarity and activation of a questioned statement favor direct retrieval (Reder, 1987). Extrinsic question factors are exemplified by task instructions, test delay, and the composition of the test materials. People can follow instructions to adopt either the retrieval or the plausibility strategy (Reder, 1982, 1987). The impact of test delay is that direct retrieval is generally favored at short test intervals, presumably because the representation constructed during learning is relatively intact. With increasing test delay, people adopt the plausibility strategy. Finally, the effect of the composition of test materials can be illustrated with reference to Set 1 , presented above. If one learns Set 1 and then has to judge whether test facts appeared in the original set, strategy selection is affected by the nature of the distractor (negative) items. In particular, if all of the distractor questions are unrelated to train travel, such as "Did Alan add bleach to the wash?" then plausibility judgments may be performed. It is unnecessary to retrieve the particular train facts that were learned. In contrast, if the distractor questions match the train context, such as "Did Alan wait for the express on the platform?" then direct retrieval must be performed. The questioned fact must be systematically compared with the learned ideas. Otherwise, one would incorrectly judge that "Did Alan wait for the express on the platform?" was a member of Set 1 .

\section{Confounding Answering Strategy and Searched Representation}

One pitfall in the analysis of answering strategies is to confound the strategy that is selected and the representation that is searched. For example, it might be assumed that direct retrieval is applied to the episodic representation of the learned material, such as a text base, whereas plausibility is evaluated with reference to pertinent world knowledge, such as the knowledge represented in a script. The temptation to confound these two dimensions is captured by the following quotations:

The static response refers to locating and returning rele-
vant information from the memory representation that was
generated at the time the text was read. A dynamic response
occurs when the relevant information is not contained in
the original memory representation but must be actively
reasoned from general world knowledge and inferencing
in conjunction with the original representation. (Lehnert,
1977, p. 57 ; italics added)

Central concepts were judged as having been present in a text more rapidly than peripheral concepts were when the test condition favored the adoption of a script-relatedness criterion for judgment. (Yekovich \& Walker, 1986, p. 635; italics added)

Lehnert's (1977) quote suggests that the static response, akin to direct retrieval, is applied to a message representation, whereas the dynamic response involves active computations based on world knowledge. Yekovich and Walker (1986) explicitly refer to a script-relatedness criterion rather than a plausibility strategy. This suggests that plausibility must be evaluated with reference to general knowledge structures, such as scripts.

However, direct retrieval is not restricted to the representation of the learned material, nor is plausibility judgment limited to structures of general knowledge. Rather, either strategy may be applied to both types of representation. For example, the question "What is the capital of Spain?" requires the retrieval of the answer from general knowledge (Camp et al., 1980). More subtle is the point that plausibility may be evaluated with reference to episodic representations. Suppose, for example, that one reads Man-Eating Tiger, a story of villagers who hired a hunter to track a tiger (Reder, 1987). If subsequently asked, "Were the villagers afraid of the tiger?" plausibility could be evaluated with reference to the message text base. It might be reasoned that because the villagers recruited a hunter, it is probable that they feared the tiger.

One factor that likely encourages the confounding of answering strategy and searched representation is that many studies of answering strategies have examined script-based materials (e.g., Reder \& Ross, 1983; Reder \& Wible, 1984; Yekovich \& Walker, 1986). In these studies, the participants have either learned facts or read texts that pertain to familiar stereotypical situations, such as going skiing or doing the laundry. In these circumstances, the direct retrieval of the questioned idea, such as "Did Susan ride the chairlift?" indeed requires a search 
of the episodic trace of the message. Plausibility, in contrast, is most effectively evaluated with reference to pertinent world knowledge, such as the skiing script.

The association of retrieval with episodic stores and plausibility judgment with world knowledge is reinforced by many studies of text recall. People's production of ideas in text recall reflects two processes: First, ideas may be reproduced from the text base. This is the recall counterpart of direct retrieval. Second, ideas may be reconstructed, an active reasoning process that operates on ideas in the text base and on pertinent knowledge structures (e.g., Hasher \& Griffin, 1978; Kintsch \& van Dijk, 1978). Reconstruction is the recall counterpart of a plausibility judgment. The analogy between recall strategies and answering strategies is reflected by the parallel impact of various factors upon strategy selection in the two tasks. For example, with increasing test delay, recall becomes increasingly reconstructive in nature (Kintsch \& van Dijk, 1978), a trend that is comparable to the shift to the plausibility strategy in delayed question answering (Reder, 1982).

Because many studies of text recall have examined script-based texts, reproduction is frequently viewed as operating on the text base, whereas reconstruction is applied to a pertinent script or schema. For example, people can reconstruct text ideas with reference to their schematic knowledge of a burglar's motives (Anderson $\&$ Pichert, 1978), of the activity of hunting (Hasher \& Griffin, 1978), of the life of Helen Keller (Sulin \& Dooling, 1974), and of the structure of a research report (Kintsch \& van Dijk, 1978). However, the correlation between recall strategy and searched representation that appears to emerge from these studies is a reflection of the script-based nature of the messages that were examined.

Not surprisingly, theoretical analyses that have been derived from studies of script-based messages sometimes reflect the spurious correlation between answering strategy and searched representation. For example, Yekovich and Walker's (1986) model of answering strategies posits plausibility to be evaluated only with reference to a pertinent script. In some cases, question-answering models substitute a stage of searched-representation selection for a stage of strategy selection (e.g., Graesser \& Franklin, 1990). This substitution carries the suggestion that the two issues are synonymous rather than orthogonal to one another.

In summary, question-answering research provides several opportunities to confound answering strategy and searched representation. First, the confounding is occasionally captured by explicit statements of researchers. Second, in numerous recognition and recall studies of answering strategies, retrieval is indeed applied to episodic knowledge structures, whereas plausibility is evaluated with reference to general knowledge. Third, a correlation between answering strategy and searched representation is implicit in some models of the memory search of question answering. A fourth, and important, difficulty is that in most, if not all, investigations, there is no direct mention of the independence of these two dimensions.

Although the potential for confounding answering strategy and searched representation is present in the literature, previous studies also provide the basis for distinguishing these two dimensions. Four types of evidence are considered in the next section: (1) examples of the application of either strategy to either episodic or general knowledge representations are provided, (2) there is experimental evidence that both direct retrieval and plausibility judgment may be applied to episodic knowledge structures, (3) there is comparable evidence of the application of both strategies to general knowledge representations, and (4) the extrinsic question factors that influence strategy selection have similar effects regardless of whether episodic or general knowledge representations are searched.

\section{Unconfounding Answering Strategy and \\ Searched Representation}

Applying the retrieval and plausibility strategies to episodic and general knowledge representations. Most analyses of answering strategies have focused on retrieval from episodic representations and judgments of plausibility with reference to general knowledge. Can each strategy be applied to the alternate representation? Languageprocessing research provides some clear examples of the opportunity to judge plausibility with reference to episodic representations. For example, Post, Greene, and Bruder's (1982) subjects read an account of an entire baseball game. They were presented with questions that probed ideas that were implied by the text. Consider the question " $\mathrm{Did}$ Winslow have at least one hit?" This question must be answered with reference to the discourse text base. The reader could evaluate the plausibility of the questioned idea by considering such factors as the frequency of mention of Winslow's name in the game transcript, the number of runs scored by Winslow's team, and the general success of the opposing pitchers. Direct retrieval could likewise be applied but would be painstaking. The announcer is unlikely to have said, "Winslow now has more than one hit." Therefore, the reader would have to consider each instance of Winslow's at bats to assess the accuracy of the questioned idea.

Likewise, Lehnert (1977) examined the story of a train ride from New York to Miami, which made no mention of the journey's duration. Consider the question "How long did the trip take?" Lehnert proposed that, because retrieval search is bound to fail, a plausibility strategy could be implemented by taking advantage of text base information, such as the fact that only one overnight was described in the episode.

As discussed earlier, Reder (1987) proposed that, after reading the Man-Eating Tiger story, people can apply the plausibility strategy to the story text base. Questions such as "Were the villagers afraid of the tiger?" and 
"Were the villagers happy that the tiger was dead?" may be judged plausible in view of the fact that the villagers recruited the hunter.

Conversely, it is even more apparent that direct retrieval may be applied to general knowledge representations. In particular, general knowledge questions such as "Who wrote 1984?" and 'Who invented dynamite?" leave little alternative but to perform direct retrieval (Camp et al., 1980).

Empirical evidence: Direct retrieval and plausibility judgment can both be applied to episodic representations. Empirical evidence that direct retrieval and plausibility judgment can both be applied to episodic representations, such as those resulting from learning a list or reading a text, stems primarily from studies of Reder (1982, 1987). Participants read stories such as Man-Eating Tiger and then answered corresponding questions. Direct retrieval had to be applied to the story text base. More importantly, plausibility likewise had to be evaluated with reference to the text base. This was because the content of the questions, such as "Were the villagers afraid of the tiger?" prevented confident plausibility judgments to be extracted on the basis of general knowledge.

Answer-time profiles of Reder's $(1982,1987)$ experiments indicated that both answering strategies were used and that they varied as a function of several factors. First, strategy was influenced by explicit task instructions. In immediate testing, people who were instructed to adopt a retrieval strategy accurately judged test items faster than did those instructed to make plausibility judgments (Reder, 1982, Table 1). Second, strategy varied with test delay. For example, the judgment times of "retrievers" were faster in immediate testing than after a 48-h delay, whereas the opposite trend was detected for those instructed to make plausibility judgments. Reder (1982) concluded that, independent of explicit instructions, people tend to shift from direct retrieval to plausibility judgment with increased test delay. Third, strategy was influenced by the composition of the test materials. For example, the participants were more likely to attempt direct retrieval when $80 \%$ of the test items explicitly appeared in the antecedent story than when only $20 \%$ of the items did (Reder, 1987, Experiment 1). Direct retrieval, of course, is effective only when a questioned statement has appeared earlier. In summary, these results indicate that both answering strategies can be applied to a story text base, an episodic representation.

Empirical evidence: Direct retrieval and plausibility judgment can both be applied to general knowledge representations. General knowledge questions, like questions about a particular message, may be answered using either the direct retrieval or the plausibility judgment strategy. As discussed earlier, the answers to some general knowledge questions cannot be computed, and so must be accessed by direct retrieval (Camp et al., 1980). This is the case whether the question takes a yes-no form, such as "Is the longest river in South America the Amazon?" or a wh-form, such as "What is the longest river in South America?"'

Other general knowledge questions, in contrast, are answered by means of plausibility judgments. These questions require certain computations or inferences before an answer can be determined. For example, "What southern (U.S.) city is named after an ocean?" (Atlanta) has been described as an inference question (Camp et al., 1980; see also Kemper, Estill, Oltavaro, \& Schadler, 1985). This is because few people are likely to have encoded the city name Atlanta as having any relation to the Atlantic Ocean. Instead, a review and evaluation of the names of major southern cities might enable the answerer to compute the correct response. Likewise, "Is the Mekong River longer than the Nile?" might be answered on the basis of a lack of knowledge computation (Gentner \& Collins, 1981). That is, one might answer "no" following the reasoning that one is not aware that the Mekong is one of the world's longest rivers. Therefore, it is unlikely that it is longer than the Nile.

To discriminate between general knowledge questions dependent on direct retrieval and those requiring inferential computations, Camp et al. (1980) presented people with questions that, on intuitive grounds, appeared to involve one of these answering strategies or the other. The subjects described their processes of answering the questions. Two judges rated those descriptions on a 5-point answer process scale. Camp et al. predicted that the direct retrieval questions would be rated toward the retrieval process end of the scale (e.g., remembering it), whereas the inferential questions would be rated as depending on computations (e.g., figuring it out). Furthermore, they predicted that the direct retrieval questions would be answered faster. The data supported these predictions. These findings suggest that both direct retrieval and plausibility judgment may be applied to general knowledge representations.

Lorch (1981) used a different procedure to show that direct retrieval and plausibility judgment can be applied to the same general knowledge store. Lorch studied the verification of property statements, such as "A robin has feathers." Like Reder (e.g., Reder, 1987; Reder \& Anderson, 1980; Reder \& Ross, 1983), Lorch manipulated the composition of his test materials. Experiment 1 included only "related," albeit unusual, false statements, such as "An apple has a fruit" (italics added). In Experiment 2 , in contrast, some additional false filler items linked two unrelated concepts, as in "A piano has a sink." Lorch proposed that the unrelated false items can be evaluated on the basis of the semantic overlap between the two concepts (i.e., piano and sink), a sort of plausibility computation. This, in turn, would favor a plausibility strategy for the entire task.

The impact of this manipulation was assessed with reference to the relatedness effect. For false statements, answer times frequently vary directly with relatedness; that is, it takes longer to verify strongly related false state- 
ments, such as "A bat is a bird," than it takes to verify weakly related ones, such as "A birch is a bird" (e.g., Collins \& Quillian, 1972; Glass, Holyoak, \& O'Dell, 1974; Rips, Shoben, \& Smith, 1973; Schaeffer \& Wallace, 1970). Table 1 presents the relatedness effects measured by Lorch (1981). A positive and significant effect of $80 \mathrm{msec}$ was detected in the presence of unrelated false filler items. Without such fillers, the relatedness effect had a negative value of $-51 \mathrm{msec}$.

This outcome supported Lorch's (1981) analysis; that is, the presence of unrelated false fillers was expected to favor the plausibility strategy, resulting in an evaluation of the semantic overlap between the two concepts of a statement. In this regard, extensive overlap between the highly related concepts apple and fruit makes it difficult and time-consuming to answer "false" to "An apple has a fruit" (cf. Smith, Shoben, \& Rips, 1974). In the absence of unrelated false fillers, in contrast, the retrieval strategy is adopted. This requires the retrieval of the subject-predicate relation linking the two concepts, resulting in longer answer times for weakly related items than for strongly related ones.

The data of an unpublished experiment (Singer, 1991) complement Lorch's (1981) findings. Concept pairs from the same category, such as "geranium tulip," were embedded in lists in which the different pairs came either from similar categories (e.g., "iris plum"- - two plants) or dissimilar categories (e.g., "iris cow"-one plant, one animal). Singer (1991) proposed the following analysis of the task: First, dissimilar different pairs form the counterpart of Lorch's (1981) unrelated false fillers. They favor the comparison of concepts by evaluating their semantic overlap, a sort of plausibility computation (Lorch, 1981; Smith et al., 1974). The similar different pairs, in contrast, require the retrieval and comparison of the pertinent category names. Second, assessing semantic overlap is less time-consuming than is the retrieval of category relations (Smith et al., 1974). Third, the strategy promoted by the different pairs is applied to all stimuli, including the same pairs. Therefore, it was predicted that judgment time for the same pairs, identical in both lists, would be faster in the dissimilar different condition than in the similar different condition.

As usual, dissimilar different pairs were judged faster than were similar different pairs (Rips et al., 1973; Schaeffer \& Wallace, 1970). The new result was that judgment time was $167 \mathrm{msec}$ faster for same pairs when they

Table 1

Relatedness Effect for False Property Statements as a Function of the Presence of Unrelated False Filler Items

\begin{tabular}{cccc}
\hline & \multicolumn{2}{c}{$\begin{array}{c}\text { Relatedness of } \\
\text { Unrelated }\end{array}$} & \multicolumn{2}{c}{$\begin{array}{c}\text { Property } \\
\text { False Fillers }\end{array}$} & Statements & & $\begin{array}{c}\text { Relatedness } \\
\text { High }\end{array}$ & Low & Effect \\
\hline Absent (Experiment 1) & 1,239 & 1,290 & -51 \\
Present (Experiment 2) & 1,319 & 1,239 & 80 \\
\hline
\end{tabular}

Note-All values are given in milliseconds. Adapted from Lorch (1981, Table 1).
Table 2

Plausibility Effect as a Function of Delay and Instructions

\begin{tabular}{lccc} 
& \multicolumn{2}{c}{ Delay } & \\
\cline { 2 - 3 } Instructions & $0 \mathrm{~min}$ & $20 \mathrm{~min}$ & Difference \\
\hline Retrieval & -22 & 90 & 112 \\
Plausibility & 115 & 266 & 151 \\
\hline
\end{tabular}

Note-All values are given in milliseconds. Adapted from Reder (1987, Table 3).

appeared in the dissimilar different list than when they appeared in the similar different list, a significant outcome. ${ }^{1}$ Effects of this sort have been interpreted as providing evidence for the impact of distractor type on answering strategy (Lorch, 1981; Reder, 1987). Singer (1991) articulated the strategy analysis of this task in terms of the two-stage model of the comparison of concept pairs (Smith et al., 1974).

In summary, the test items examined by Camp et al. (1980), Lorch (1981), and Singer (1991) could be evaluated only with reference to semantic memory. Therefore, their data support the proposal that both direct retrieval and plausibility judgment may be applied to the same knowledge representation. That, in turn, supports the proposal that answering strategy is independent of the searched representation.

Parallel effects of strategy factors in the search of episodic and general knowledge representations. This section reviews evidence that extrinsic question factors exert parallel effects on strategy selection regardless of whether plausibility is evaluated with reference to episodic or general knowledge structures. Consider first the joint effect of test delay and task instructions. Reder's (1987) examination of stories such as Man-Eating Tiger favored the evaluation of plausibility with reference to the story text base. One index of strategy choice is whether it takes longer to answer questions of moderate plausibility than to answer questions of high plausibility. This effect ought to appear only if one performs plausibility judgments.

Table 2 shows the plausibility effect as a function of instruction (retrieval vs. plausibility) and test delay ( 0 vs. $20 \mathrm{~min}$ ) (Reder, 1987, Experiment 2). The plausibility effect increased as a function of test delay by $112 \mathrm{msec}$ in the retrieval condition and $151 \mathrm{msec}$ in the plausibility condition. This outcome reflects people's preference for plausibility judgment with increased delay.

A parallel profile is observed when plausibility must be evaluated with reference to world knowledge. The participants of Reder and Wible (1984), for example, learned zero, two, or four facts that related character names to various themes. For example, a participant could learn two facts about a train journey, such as "Alan bought a ticket for the 10:00 train" and "Alan heard the conductor call, 'All aboard." ' A fact retrieval test was administered either immediately or 2 days later, and the participants received either retrieval or plausibility instructions.

The index of strategy choice in this task was the size of the fan effect. The classic fan effect refers to the observation that answer time increases systematically with 
Table 3

Composite Fan Effect for Stated and Inconsistent Items as a Function of Delay and Instructions

\begin{tabular}{|c|c|c|c|}
\hline \multirow[b]{2}{*}{ Instructions } & \multicolumn{2}{|c|}{ Delay } & \multirow[b]{2}{*}{ Difference } \\
\hline & $0 \mathrm{~min}$ & 2 days & \\
\hline Retrieval & 135 & -65 & -200 \\
\hline Plausibility & 12 & -146 & -158 \\
\hline
\end{tabular}

Note-All values are given in milliseconds. Adapted from Reder and Wible (1984).

the number of facts known about the questioned concept (e.g., Anderson, 1976). However, a positive fan effect is observed only under the retrieval strategy. When one makes plausibility judgments, the fan effect is either negligible or negative (Reder \& Anderson, 1980). Table 3 presents the composite fan effect for stated and inconsistent test items (Reder \& Wible, 1984). With increased delay, the fan effect became $200 \mathrm{msec}$ more negative in the retrieval instruction condition and $158 \mathrm{msec}$ more negative in the plausibility condition. That is, in both instruction groups, people's reliance on the plausibility strategy increased with test delay. This parallels the shift to plausibility judgment previously examined in Table 2 . In conclusion, people shift toward plausibility judgments with increased delay, regardless of whether plausibility is evaluated with reference to episodic or general knowledge structures.

Consider next the effect of test material composition. As discussed earlier, Reder (1987, Experiment 1) varied the percentage of plausible test items that had been presented explicitly in antecedent stories: $80 \%$ or $20 \%$. The $80 \%$-presented and the $20 \%$-presented conditions were expected to favor the retrieval and plausibility strategies, respectively. The difference between judgment time for items of high and moderate plausibility, the plausibility effect, supported this prediction. The plausibility effect in the $80 \%$ condition was $75 \mathrm{msec}$, a negligible value. In the $20 \%$-stated condition, in contrast, the plausibility effect was $445 \mathrm{msec}$. The stories, such as Man-Eating Tiger, favored the assessment of plausibility with reference to the story text base. Therefore, composition of test materials can influence strategy selection when plausibility is evaluated with reference to a text base representation.

Composition of test materials can likewise affect strategy selection when plausibility is assessed on the basis of general knowledge. The participants of Reder and Anderson (1980) learned sets of either one or three facts related to such themes as train travel. Example Set 1 is repeated here.

1. a. Alan arrived at the train station early.

b. Alan bought a ticket for the 10:00 train.

c. Alan heard the conductor call, "All aboard."

Test blocks presented distractor items that either were unrelated to the learned set, such as "Alan added bleach to the wash," or were related, such as "Alan waited for the express on the platform." Answer times revealed a fan effect of $123 \mathrm{msec}$ in the related distractor condition, compared with a negligible effect of $20 \mathrm{msec}$ in the unrelated distractor condition (Reder \& Anderson, 1980, Experiment 1). As discussed earlier, a positive fan effect reflects use of direct retrieval, whereas a negligible fan effect suggests the adoption of a plausibility strategy. Thus, composition of test materials can influence strategy selection, regardless of whether plausibility is assessed against a message text base or pertinent world knowledge. In conclusion, extrinsic question factors have similar effects on strategy selection, regardless of whether plausibility is evaluated with reference to episodic representations or to general knowledge structures. This supports the proposal that answering strategy is independent of searched representation.

\section{CONCLUSION}

In this paper, it has been proposed that strategy selection in question answering is independent of the representation to which a strategy is applied. However, there has been the tendency to assume that direct retrieval is applied to episodic structures, such as message text bases, whereas plausibility is applied to general knowledge structures, such as scripts. Four factors that contribute to this confounding were identified: (1) the literature includes explicit examples of the confounding, (2) the confounding is promoted by the fact that many studies of answering strategy have examined script-based messages, (3) some models derived from the latter studies incorporate the confounding, and (4) theorists have not explicitly identified the independence of answering strategy and searched representation.

In support of the independence hypothesis, several types of evidence were considered. First, both previous research and intuition provide examples of the application of direct retrieval to general knowledge and of plausibility strategies to episodic representations. Second, evidence showing that either strategy can be applied to either type of representation was reviewed. Finally, the extrinsic factors that influence strategy selection were shown to exert similar effects in the search of episodic and general knowledge representations.

The present analysis implies that the selection mechanisms for answering strategies and those for the searched representation will need to be addressed independently. Reder (1987) proposed that strategy selection is influenced by an automatic evaluation of the familiarity and activation of the questioned idea and that this evaluation may, in turn, contribute to controlled processes of strategy selection. The mechanisms for the selection of the searched representation may likewise include automatic and controlled components. For example, Schmalhofer and Glavanov (1986) reported that sentence recognition varied directly with the number of levels of text representation (form, text base, and situation model) with which the test sentence was consistent. It is speculated that each of these representations supported recognition automati- 
cally and that the participants did not systematically compare the question with alternate representations. This proposal is consistent with a construction-integration analysis of sentence memory (Kintsch, 1988; Kintsch, Welsch, Schmalhofer, \& Zimny, 1990). According to constructionintegration, a test question is compared with one composite trace of the entire antecedent discourse. In certain circumstances, such as delayed testing, answer retrieval is predominantly influenced by the situation model component of the composite trace, a component which may include pertinent script information (Kintsch et al., 1990).

In contrast, Kolodner (1983) described a hypothetical controlled process whereby the answerer selects a candidate representation to search. She presented the example of a government official who is asked when he last saw an oil field in the Middle East. Kolodner proposed that, in the event of retrieval failure, the official might focus on the more constrained category of "diplomatic visits to the Middle East," following the logic that he would not likely have visited an oil field on a vacation trip.

Another issue requiring further examination is the evaluation of plausibility with reference to message text bases. Two problems merit consideration. First, it is unlikely that plausibility can be evaluated purely with reference to a text base. For example, to evaluate the plausibility of "The villagers were afraid of the tiger" (Reder, 1982, 1987), the understander may invoke the general knowledge that villagers usually fear tigers, in addition to text base ideas. Likewise, to answer "How long did the trip take?" with reference to Lehnert's (1977) train journey story, plausibility judgment could take into account knowledge of the 1,300 mile distance between New York and Miami. Therefore, it will be necessary to address the interaction of episodic and general knowledge in judging plausibility with reference to complex messages.

Second, an alternate explanation of how Reder's (1987) participants answered "Were the villagers afraid of the tiger?" is that the villagers' fear of the tiger may have acquired the status of a text macroproposition, on the basis of the generalization of text ideas (e.g., Kintsch \& Young, 1984). In that event, the test statement would have been directly retrievable from the text base.

In addition to having to address the cognitive mechanisms of strategy selection and representation selection, theorists will need to grapple with the interactions between these mechanisms. This prospect is qualitatively similar to the problems of the interactions among other stages of question answering-namely, daunting but unavoidable.

\section{REFERENCES}

ANDERSON, J. R. (1976). Language, memory, and thought. Hillsdale, NJ: Erlbaum.

Anderson, R. C., \& Pichert, J. W. (1978). Recall of previously unrecallable information following a shift in perspective. Journal of Verbal Learning \& Verbal Behavior, 17, 1-12.

Camp, C. J., Lachman, J. L., \& Lachman, R. (1980). Evidence for direct-access and inferential retrieval in question-answering. Journal of Verbal Learning \& Verbal Behavior, 19, 583-596.
Coll.ins, A. M., \& Quil.lian, M. R. (1972). Experiments on semantic memory and language comprehension. In L. Gregg (Ed.), Cognition in learning and memory (pp. 117-137). New York: Wiley.

Galambos, J. A. B Back, J. B. (1985). Using knowledge of activities to understand and answer questions. In A. Graesser \& J. Black (Eds.). The psychology of questions (pp. 157-189). Hillsdale. NJ: Erlbaum.

Gentner, D. Collins, A. (1981). Studies of inference from lack of knowledge. Memory \& Cognition, 9, 434-443

Glass, A. L., Holyoak, K. J., \& O'Dell, C. (1974). Production frequency and the verification of quantified statements. Journal of Verbal Learning \& Verbal Behavior, 13, 237-254

Graesser, A. C., \& Franklin, S. P. (1990). QUEST: A cognitive model of question answering. Discourse Processes, 13, 279-303.

Graesser, A. C., Murachver, T. (1985). Symbolic procedures of question answering. In A. Graesser \& J. Black. The psychology of questions (pp. 15-88). Hillsdale, NJ: Erlbaum.

Graesser, A. C., Robertson, S. P.. Lovelace, E. R.. \& SwineHART, D. M. (1980). Answers to why-questions expose the organization of story plot and predict recall of actions. Journal of Verbal Learning \& Verbal Behavior, 19, 110-119.

Hasher, L.. \& GRIFFIN, M. (1978). Reconstructive and reproductive processes in memory. Journal of Experimental Psychology: Human Learning \& Memory, 4, 318-330.

Kemper, S., Estill, R., Oltavaro, N., \& Schadler, M. (1985). Questions of facts and questions of inferences. In A. Graesser \& J. Black (Eds.). The psychology of questions (pp. 227-246). Hillsdale. NJ Erlbaum.

KINTSCH, W. (1988). The role of knowledge in discourse comprehension: A construction-integration model. Psychological Review, 95 , 163-182.

KinTsCh, W., \& VAN Duk, T. A. (1978). Toward a model of text comprehension and production. Psychological Review, 85, 363-394.

Kintsch, W., Welsch, D., Schmalhofer, F., \& ZimnY, S. (1990). Sentence memory: A theoretical analysis. Joumal of Memory \& Language. 29, 133-159

KINTSCH, W., \& YoUNG, S. R. (1984). Selective recall of decisionrelevant information from texts. Memory \& Cognition, 12, 112-117.

Kolodner, J. L. (1983). Reconstructive memory: A computer model. Cognitive Science, 7, 281-328.

LEHNERT, W. (1977). Human computational question answering. Cognitive Science, 1, 47-73

LoRCH, R. F., JR. (1981). Effects of relation strength and semantic overlap on retrieval and comparison processes during sentence verification. Journal of Verbal Learning \& Verbal Behavior, 20, 593-611.

Post, T. A., Greene, T., \& Bruder, G. (1982, November). "On-line" text processing in high-and low-knowledge individuals. Paper presented at the annual meeting of the Psychonomic Society, Minneapolis, $\mathrm{MN}$

REDER, L. M. (1982). Plausibility judgments versus fact retrieval: Alternative strategies for sentence verification. Psychological Review. 89, 250-280.

REDER, L. M. (1987). Strategy-selection in question answering. Cog nitive Psychology, 19, 90-134.

REDER, L. M., \& ANDERSON, J. R. (1980). A partial resolution of the paradox of interference: The role of integrating knowledge. Cognitive Psychology, 12, 447-472.

REDER, L. M., \& Ross, B. H. (1983). Integrated knowledge in different tasks: The role of retrieval strategy on fan effects. Journal of $E x$ perimental Psychology: Learning, Memory, \& Cognition, 9, 55-72.

Reder, L. M., \& WBLE, C. (1984). Strategy use in question answering: Memory strength and task constraints on fan effects. Memory \& Cognition, 12, 411-419.

Rips, L. J., Shoben, E. J., \& Sмith, E. E. (1973). Semantic distance and the verification of semantic relations. Journal of Verbal Learning \& Verbal Behavior, 12, 1.20.

SCHAEFFER, B., WALLACE, R. (1970). The comparison of word meanings. Journal of Experimental Psychology, 86, 144-152.

Schmalhofer. F., Glavanov, D. (1986). Three components of understanding a programmer's manual: Verbatim, propositional, and 
situation representations. Journal of Memory \& Language, 25, 279-294.

Singer, M. (1986). Answering wh- questions about sentences and text. Journal of Memory \& Language, 25, 238-254.

SINGER, M. (1991). Question-answering strategies and conceptual knowledge. Bulletin of the Psychonomic Society, 29, 143-146.

Smith, E. E., Shoben, E. J., \& Rips, L. J. (1974). Structure and process in semantic memory: A feature model of semantic decisions. Psychological Review, 81, 214-241.

Sulin, R. A., \& Dooling, D. J. (1974). Intrusion of a thematic idea in retention of prose. Journal of Experimental Psychology, 103, 255-262.

Yekovich, F. R., \& WALKER, C. H. (1986). Retrieval of scripted concepts. Journal of Memory \& Language, 25, 627-644.

\section{NOTE}

1. In the experiment, 120 native-English-speaking introductory psychology students viewed either the similar different list or the dissimilar different list. Each list included 36 same pairs and 36 different pairs. All statistical tests used the criterion of $\alpha=.05$, and were executed altemately treating subjects and stimuli as the random variable.

(Manuscript received July 2, 1990; revision accepted for publication September 10, 1990.)

\title{
Announcement
}

\author{
Second International Symposium \\ on Memory and Awareness in Anesthesia \\ Atlanta, Georgia \\ April 23-25, 1992
}

\section{CALL FOR ABSTRACTS}

The Department of Anesthesiology and the Department of Psychology of Emory University are pleased to announce that the Second International Symposium on Memory and Awareness in Anesthesia will be held at the Hotel Nikko, Atlanta, April 23-25, 1992.

Abstracts are invited on the following topics: Awareness in general anesthesia, including definition, occurrence/incidence, and causes; memory for intraoperative events; effects of suggestion; information processing in the unconscious mind; memory and awareness in relation to anesthetics used; techniques for monitoring the nervous system.

Abstracts, preferably one page single spaced, should be sent to one of the members of the organizing committee: Eugene Winograd, Department of Psychology, Emory University, Atlanta, GA 30322; Peter Sebel, Department of Anesthesiology, Crawford Long Hospital, Glenn Building, 25 Prescott Street, N.E., Atlanta, GA 30308; or Benno Bonke, Department of Medical Psychology, Faculty of Medicine, Erasmus University, Rotterdam, The Netherlands. The deadline for receipt of the abstracts is November 1, 1991.

For registration information, write to Susan J. Duensing, Continuing Medical Education Program Director, Emory University School of Medicine, 1440 Clifton Road, N.E., 109 WHSCAB, Atlanta, GA 30322. 